PROCEEDINGS OF THE

AMERICAN MATHEMATICAL SOCIETY

Volume 139, Number 5, May 2011, Pages 1553-1567

S 0002-9939(2010)10585-3

Article electronically published on September 16, 2010

\title{
HOCHSCHILD COHOMOLOGY OF GROUP EXTENSIONS OF QUANTUM SYMMETRIC ALGEBRAS
}

\author{
DEEPAK NAIDU, PIYUSH SHROFF, AND SARAH WITHERSPOON
}

(Communicated by Martin Lorenz)

\begin{abstract}
Quantum symmetric algebras (or noncommutative polynomial rings) arise in many places in mathematics. In this article we find the multiplicative structure of their Hochschild cohomology when the coefficients are in an arbitrary bimodule algebra. When this bimodule algebra is a finite group extension (under a diagonal action) of a quantum symmetric algebra, we give explicitly the graded vector space structure. This yields a complete description of the Hochschild cohomology ring of the corresponding skew group algebra.
\end{abstract}

\section{INTRODUCTION}

The action of a group $G$ on a vector space $V$ induces an action on the corresponding symmetric algebra $S(V)$ (a polynomial ring). The resulting skew group algebra $S(V) \rtimes G$ is a noncommutative ring encoding the group action. Deformations of such skew group algebras are related to deformations of corresponding orbifolds, and some have appeared independently in several places under different names: graded Hecke algebras, rational Cherednik algebras, and symplectic reflection algebras. Deformations of any algebra are intimately related to its Hochschild cohomology. When $G$ is finite the Hochschild cohomology of $S(V) \rtimes G$ was computed independently by Farinati $\mathrm{F}$ and by Ginzburg and Kaledin GK. Its algebra structure was first given by Anno [A].

In this paper we replace the symmetric algebra $S(V)$ with a quantum symmetric algebra and explore its Hochschild cohomology. Our quantum symmetric algebra is a noncommutative polynomial ring, denoted $S_{\mathbf{q}}(V)$, in which the variables commute only up to multiplication by nonzero scalars (encoded in the vector $\mathbf{q}$ ). Noncommutative polynomials have been of interest for some time. Our current work incorporates group actions as well and is in part motivated by the recent appearance of two articles. First, Kirkman, Kuzmanovich, and Zhang KKZ prove a version of the classical Shephard-Todd-Chevalley Theorem, namely that the invariant subring of $S_{\mathbf{q}}(V)$ under a finite group action is again a quantum symmetric algebra. In the setting of an ordinary polynomial ring, methods from invariant theory for finding such subrings play a crucial role in computation of Hochschild cohomology (see, for

Received by the editors November 19, 2009 and, in revised form, May 14, 2010.

2010 Mathematics Subject Classification. Primary 16E40, 16S35.

The second and third authors were partially supported by NSF grant \#DMS-0800832 and Advanced Research Program Grant 010366-0046-2007 from the Texas Higher Education Coordinating Board.

(C)2010 American Mathematical Society

Reverts to public domain 28 years from publication 
example, $[\mathrm{SW}]$ ). Around the same time, Bazlov and Berenstein $\mathrm{BB}$ introduced braided Cherednik algebras, which are deformations of $S_{\mathbf{q}}(V) \rtimes G$ in some special cases. Knowledge of Hochschild cohomology will provide insight into these and other possible deformations.

More specifically, let $\mathbb{k}$ be a field of characteristic 0 . Let $N$ be a positive integer and for each pair $i, j$ of elements in $\{1, \ldots, N\}$, let $q_{i, j}$ be a nonzero scalar such that $q_{i, i}=1$ and $q_{j, i}=q_{i, j}^{-1}$ for all $i, j$. Denote by $\mathbf{q}$ the corresponding tuple of scalars, $\mathbf{q}:=\left(q_{i, j}\right)_{1 \leq i<j \leq N}$. Let $V$ be a vector space with basis $x_{1}, \ldots, x_{N}$, and let

$$
\left.S_{\mathbf{q}}(V):=\mathbb{k}\left\langle x_{1}, \ldots, x_{N}\right| x_{i} x_{j}=q_{i, j} x_{j} x_{i} \text { for all } 1 \leq i, j \leq N\right\rangle,
$$

the quantum symmetric algebra determined by q. This is a Koszul algebra (see e.g. [AS], so there is a standard complex $K \cdot\left(S_{\mathbf{q}}(V)\right)$ that is a resolution of $S_{\mathbf{q}}(V)$ as an $S_{\mathbf{q}}(V)$-bimodule. This complex is given in Section 2.1 below; see [W] for details on more general quantum symmetric algebras arising from braidings. Priddy $[\mathrm{P}]$ first introduced Koszul algebras, and the theory was developed further, including such complexes, in BGS, BG, $\mathrm{M}$.

We first compute cup products, using the resolution in Section 2.1, and obtain the following theorem. Here $\bigwedge_{\mathbf{q}^{-1}}\left(V^{*}\right)$ denotes a quantum exterior algebra, defined in (9) below, on the dual vector space $V^{*}$, and $B$ is any algebra with a compatible structure of an $S_{\mathbf{q}}(V)$-bimodule. The two choices of algebra $B$ in which we are most interested are $B=S_{\mathbf{q}}(V)$ and $B=S_{\mathbf{q}}(V) \rtimes G$, where $G$ is a finite group of graded automorphisms of $S_{\mathbf{q}}(V)$.

Theorem 3.1. The Hochschild cohomology $\mathrm{HH}^{*}\left(S_{\mathbf{q}}(V), B\right)$ is a subquotient algebra of the tensor product $B \otimes \bigwedge_{\mathbf{q}^{-1}}\left(V^{*}\right)$.

That is, the Hochschild cohomology is a vector subquotient of $B \otimes \bigwedge_{\mathbf{q}^{-1}}\left(V^{*}\right)$, and its cup product is determined by that of the tensor product of the two algebras $B$ and $\bigwedge_{\mathbf{q}^{-1}}\left(V^{*}\right)$.

We next give the graded vector space structure of Hochschild cohomology in the two cases $B=S_{\mathbf{q}}(V)$ and $B=S_{\mathbf{q}}(V) \rtimes G$, when $G$ acts diagonally on the basis $x_{1}, \ldots, x_{N}$ of $V$. We adapt techniques developed in the context of Hochschild homology by Wambst [W]. In the first case this gives the following technical result. The notation will be explained in Section 4 .

Corollary 4.3. For all $m \in \mathbb{N}$,

$$
\operatorname{HH}^{m}\left(S_{\mathbf{q}}(V)\right) \cong \bigoplus_{\substack{\beta \in\{0,1\}^{N} \\|\beta|=m}} \bigoplus_{\substack{\alpha \in \mathbb{N}^{N} \\ \alpha-\beta \in C}} \operatorname{span}_{\mathbb{k}}\left\{x^{\alpha} \otimes\left(x^{*}\right)^{\wedge \beta}\right\} .
$$

This result is a consequence of our more general Theorem 4.1 on $S_{\mathbf{q}}(V) \rtimes G$. It should be compared with work of Richard $[\mathrm{R}$, in which there are some results on the Hochschild cohomology of a related ring of twisted differential operators on quantum affine space. Richard obtains his results by first computing Hochschild homology and then invoking a duality between homology and cohomology. In our setting, such a duality does not hold in general; a comparison of our Example 4.2 below with Wambst's Corollary $6.2[\mathrm{~W}]$ shows that there is no duality in our smallest possible case. Other authors have used various techniques to compute Hochschild homology of generalizations of $S_{\mathbf{q}}(V)$ GG, Gu, W]. 
Since the characteristic of $\mathbb{k}$ is 0 , the Hochschild cohomology of $S_{\mathbf{q}}(V) \rtimes G$ is the subalgebra of $G$-invariant elements of $\operatorname{HH}^{*}\left(S_{\mathbf{q}}(V), S_{\mathbf{q}}(V) \rtimes G\right)$, and the multiplicative structure of this algebra is given by Theorem 3.1. To determine the additive structure precisely, we specialize to diagonal actions of $G$ on the chosen basis $x_{1}, \ldots, x_{N}$ of $V$. This is the case to which Wambst's techniques in W] may be most easily adapted to express the relevant cochain complex as the direct sum of an acyclic complex and a complex in which all differentials are 0 . This leads to our description of the Hochschild cohomology as a graded vector space in the following theorem. The notation will be explained in Section 4

Theorem 4.1. Assume that the finite group $G$ acts diagonally on the chosen basis of $V$. Then for all $m \in \mathbb{N}$,

$$
\operatorname{HH}^{m}\left(S_{\mathbf{q}}(V), S_{\mathbf{q}}(V) \rtimes G\right) \cong \bigoplus_{g \in G} \bigoplus_{\substack{\beta \in\{0,1\}^{N} \\|\beta|=m}} \bigoplus_{\substack{\alpha \in \mathbb{N}^{N} \\ \alpha-\beta \in C_{g}}} \operatorname{span}_{\mathbb{k}}\left\{\left(x^{\alpha} \# g\right) \otimes\left(x^{*}\right)^{\wedge \beta}\right\},
$$

and $\mathrm{HH}^{m}\left(S_{\mathbf{q}}(V) \rtimes G\right)$ is its $G$-invariant subspace.

We plan to address more general group actions, as well as related deformations of the skew group algebra $S_{\mathbf{q}}(V) \rtimes G$, in future articles.

Organization. This paper is organized as follows. Section 2 contains necessary preliminary information, including the resolution of $S_{\mathbf{q}}(V)$ that will be used. We also give a chain map from this resolution to the bar resolution of $S_{\mathbf{q}}(V)$, used in Section 3 to compute cup products.

We prove Theorem 3.1 in Section 3. In Section 4 we prove Theorem 4.1 and apply Theorem 3.1 to give the cup product on $\operatorname{HH}^{*}\left(S_{\mathbf{q}}(V) \rtimes G\right)$. As a special case, when $G=\{1\}$, we obtain in Corollary 4.3 the Hochschild cohomology of $S_{\mathbf{q}}(V)$.

\section{Preliminaries}

All tensor products and exterior powers are taken over the field $\mathbb{k}$ of characteristic 0 .

Let $A$ be an algebra over $\mathbb{k}$, and let $M$ be an $A$-bimodule. We identify $M$ with a (left) $A^{e}$-module, where $A^{e}=A \otimes A^{\mathrm{op}} ; A^{\mathrm{op}}$ is the algebra $A$ with the opposite multiplication. The Hochschild cohomology of $A$ with coefficients in $M$ is

$$
\operatorname{HH}^{*}(A, M):=\operatorname{Ext}_{A^{e}}(A, M),
$$

where $A$ is considered to be an $A^{e}$-module under left and right multiplication. One useful free $A^{e}$-resolution of $A$ is the bar resolution

$$
\text { ‥ } \stackrel{\delta_{3}}{\longrightarrow} A^{\otimes 4} \stackrel{\delta_{2}}{\longrightarrow} A^{\otimes 3} \stackrel{\delta_{1}}{\longrightarrow} A^{e} \stackrel{\text { mult }}{\longrightarrow} A \rightarrow 0,
$$

where $\delta_{m}\left(a_{0} \otimes \cdots \otimes a_{m+1}\right)=\sum_{i=0}^{m}(-1)^{i} a_{0} \otimes \cdots \otimes a_{i} a_{i+1} \otimes \cdots \otimes a_{m+1}$ for all $a_{0}, \ldots, a_{m+1} \in A$, and the map from $A^{e}$ to $A$ is given by multiplication in $A$. Suppose $M=B$ is an $A$-bimodule algebra, that is $B$ is an algebra and also an $A$-bimodule and that these two structures are compatible in the sense that $a\left(b b^{\prime}\right)=$ $(a b) b^{\prime}$ and $\left(b b^{\prime}\right) a=b\left(b^{\prime} a\right)$ for all $a \in A$ and $b, b^{\prime} \in B$. Then $\operatorname{HH}^{*}(A, B)$ has a cup product defined at the cochain level as follows (e.g. see [G. p. 278]). Let $f \in$ $\operatorname{Hom}_{A^{e}}\left(A^{\otimes(m+2)}, B\right), f^{\prime} \in \operatorname{Hom}_{A^{e}}\left(A^{(n+2)}, B\right)$. Then $f \smile f^{\prime} \in \operatorname{Hom}_{A^{e}}\left(A^{\otimes(m+n+2)}, B\right)$ 
is determined by

$$
\begin{aligned}
f & \smile f^{\prime}\left(a_{0} \otimes a_{1} \otimes \cdots \otimes a_{m+n} \otimes a_{m+n+1}\right) \\
& =f\left(a_{0} \otimes a_{1} \otimes \cdots \otimes a_{m} \otimes 1\right) f^{\prime}\left(1 \otimes a_{m+1} \otimes \cdots \otimes a_{m+n} \otimes a_{m+n+1}\right) .
\end{aligned}
$$

Let $G$ be a finite group acting on the algebra $A$ by automorphisms. We denote by ${ }^{g} a$ the result of applying $g \in G$ to $a \in A$. Then we may form the skew group algebra $A \rtimes G$ : Additively, it is the free $A$-module with basis $G$. We write $A \rtimes G=\bigoplus_{g \in G} A_{g}$, where $A_{g}=\{a \# g \mid a \in A\}$; that is, for each $a \in A$ and $g \in G$ we denote by $a \# g \in A_{g}$ the $a$-multiple of $g$. Multiplication on $A \rtimes G$ is determined by

$$
(a \# g)(b \# h):=a\left({ }^{g} b\right) \# g h
$$

for all $a, b \in A, g, h \in G$. Note that for each $g \in G, A_{g}$ is a (left) $A^{e}$-module via the action

$$
(a \otimes b) \cdot(c \# g):=(a \# 1)(c \# g)(b \# 1)=a c\left({ }^{g} b\right) \# g
$$

for all $a, b, c \in A, g \in G$.

For convenience in what follows, we will sometimes denote the quantum symmetric algebra $S_{\mathbf{q}}(V)$ simply by $A$.

2.1. A free resolution of $S_{\mathbf{q}}(V)$. By [W] Proposition 4.1(c)], the following is a free $A^{e}$-resolution of $A=S_{\mathbf{q}}(V)$ :

$$
\cdots \rightarrow A^{e} \otimes \bigwedge^{2}(V) \stackrel{d_{2}}{\longrightarrow} A^{e} \otimes \bigwedge^{1}(V) \stackrel{d_{1}}{\longrightarrow} A^{e} \stackrel{\text { mult }}{\longrightarrow} A \rightarrow 0 .
$$

That is, for $1 \leq m \leq N$, the degree $m$ term is $A^{e} \otimes \bigwedge^{m}(V)$, and $d_{m}$ is defined by

$$
\begin{aligned}
& d_{m}\left(1^{\otimes 2} \otimes x_{j_{1}} \wedge \cdots \wedge x_{j_{m}}\right) \\
& =\sum_{i=1}^{m}(-1)^{i+1}\left[\left(\prod_{s=1}^{i} q_{j_{s}, j_{i}}\right) x_{j_{i}} \otimes 1-\left(\prod_{s=i}^{m} q_{j_{i}, j_{s}}\right) \otimes x_{j_{i}}\right] \otimes x_{j_{1}} \wedge \cdots \wedge \hat{x}_{j_{i}} \wedge \cdots \wedge x_{j_{m}}
\end{aligned}
$$

whenever $1 \leq j_{1}<\cdots<j_{m} \leq N$. This is a twisted version of the usual Koszul resolution for a polynomial ring.

Let us write the above formula for $d_{m}$ in a more convenient form. We first introduce some notation following Wambst $\mathrm{W}$. Let $\mathbb{N}^{N}$ denote the set of all $N$ tuples of elements from $\mathbb{N}$. For any $\alpha \in \mathbb{N}^{N}$, the length of $\alpha$, denoted $|\alpha|$, is the sum $\sum_{i=1}^{N} \alpha_{i}$. For all $\alpha \in \mathbb{N}^{N}$, define $x^{\alpha}:=x_{1}^{\alpha_{1}} x_{2}^{\alpha_{2}} \cdots x_{N}^{\alpha_{N}}$. For all $i \in\{1, \ldots, N\}$, define $[i] \in \mathbb{N}^{N}$ by $[i]_{j}=\delta_{i, j}$, for all $j \in\{1, \ldots, N\}$. For any $\beta \in\{0,1\}^{N}$, let $x^{\wedge \beta}$ denote the vector $x_{j_{1}} \wedge \cdots \wedge x_{j_{m}} \in \Lambda^{m}(V)$ which is defined by $m=|\beta|, \beta_{j_{k}}=1$ for all $k \in\{1, \ldots, m\}$, and $j_{1}<\cdots<j_{m}$. Then, for any $\beta \in\{0,1\}^{N}$ with $|\beta|=m$ we have

$$
\begin{aligned}
d_{m}\left(1^{\otimes 2} \otimes x^{\wedge \beta}\right)= & \sum_{i=1}^{N} \delta_{\beta_{i}, 1}(-1)^{\sum_{s=1}^{i-1} \beta_{s}}\left[\left(\prod_{s=1}^{i} q_{s, i}^{\beta_{s}}\right) x_{i} \otimes 1-\left(\prod_{s=i}^{N} q_{i, s}^{\beta_{s}}\right) \otimes x_{i}\right] \\
& \otimes x^{\wedge(\beta-[i])} .
\end{aligned}
$$

2.2. A chain map into the bar resolution of $S_{\mathbf{q}}(V)$. We wish to define a chain map from our complex $A^{e} \otimes \bigwedge^{\circ}(V)$ to the bar resolution (1) for $A=S_{\mathbf{q}}(V)$. Wambst defined a more general chain map [W, Lemma 5.3 and Theorem 5.4]. Here we introduce notation useful in our setting, and include some details for completeness. 
For each set of $m$ distinct natural numbers $j_{1}, \ldots, j_{m}(m \leq N)$ and each permutation $\pi \in S_{m}$, the scalar $q_{\pi}^{j_{1}, \ldots, j_{m}}$ is defined by the equation

$$
q_{\pi}^{j_{1}, \ldots, j_{m}} x_{j_{\pi(1)}} \cdots x_{j_{\pi(m)}}=x_{j_{1}} \cdots x_{j_{m}}
$$

in $A$. That is, $q_{\pi}^{j_{1}, \ldots, j_{m}}$ is the scalar arising when one applies the permutation $\pi$ to the variables in the product $x_{j_{1}} \cdots x_{j_{m}}$, using the relations in $A$ to rewrite it.

The following lemma is immediate from the definition.

Lemma 2.1. If $\pi=\sigma \tau$ in $S_{m}$, then

$$
q_{\pi}^{j_{1}, \ldots, j_{m}}=q_{\sigma}^{j_{\tau(1)}, \ldots, j_{\tau(m)}} q_{\tau}^{j_{1}, \ldots, j_{m}} .
$$

For each $m \geq 1$, define the map $\phi_{m}: A^{e} \otimes \bigwedge^{m}(V) \rightarrow A^{\otimes(m+2)}$ by

$$
\phi_{m}\left(1^{\otimes 2} \otimes x_{j_{1}} \wedge \cdots \wedge x_{j_{m}}\right)=\sum_{\pi \in S_{m}}(\operatorname{sgn} \pi) q_{\pi}^{j_{1}, \ldots, j_{m}} \otimes x_{j_{\pi(1)}} \otimes \cdots \otimes x_{j_{\pi(m)}} \otimes 1
$$

for all distinct $x_{j_{1}}, \ldots, x_{j_{m}}$. Note that $\phi_{m}$ is injective: The image of a basis of $\bigwedge^{m}(V)$ under $\phi_{m}$ is linearly independent, as may be seen by comparing the variables involved. Set $\phi_{0}$ and $\phi_{-1}$ to be the identity maps on $A \otimes A$ and $A$, respectively.

Remark 2.2. By its definition, the image of $\phi_{m}$ is contained in

$$
\bigcap_{i=0}^{m-2}\left(A \otimes V^{\otimes i} \otimes R \otimes V^{\otimes(m-i-2)} \otimes A\right),
$$

where $R \subset V \otimes V$ is the vector subspace spanned by the relations $x_{i} \otimes x_{j}-q_{i, j} x_{j} \otimes x_{i}$. For example, to see that $\phi_{m}\left(1^{\otimes 2} \otimes x_{j_{1}} \wedge \cdots \wedge x_{j_{m}}\right)$ is in $A \otimes R \otimes V^{\otimes(m-2)} \otimes A$, fix $\pi \in S_{m}$. Let (12) denote the permutation transposing 1 and 2 . The $\pi$ - and $\pi(12)$ terms of formula (3) above are (writing $\pi(12)=(\pi(1), \pi(2)) \pi$ in $S_{m}$ and applying the lemma):

$$
\begin{aligned}
& \quad(\operatorname{sgn} \pi) q_{\pi}^{j_{1}, \ldots, j_{m}} \otimes x_{j_{\pi(1)}} \otimes \cdots \otimes x_{j_{\pi(m)}} \otimes 1+(\operatorname{sgn} \pi(12)) q_{\pi(12)}^{j_{1}, \ldots, j_{m}} \\
& \quad \otimes x_{j_{\pi(2)}} \otimes x_{j_{\pi(1)}} \otimes \cdots \otimes x_{j_{\pi(m)}} \otimes 1 \\
& =(\operatorname{sgn} \pi) q_{\pi}^{j_{1}, \ldots, j_{m}} \otimes x_{j_{\pi(1)}} \otimes \cdots \otimes x_{j_{\pi(m)}} \otimes 1 \\
& \quad-(\operatorname{sgn} \pi) q_{(\pi(1), \pi(2))}^{j_{\pi(1)}, \ldots, j_{\pi(m)}} q_{\pi}^{j_{1}, \ldots, j_{m}} \otimes x_{j_{\pi(2)}} \otimes x_{j_{\pi(1)}} \otimes \cdots \otimes x_{j_{\pi(m)}} \otimes 1 \\
& =(\operatorname{sgn} \pi) q_{\pi}^{j_{1}, \ldots, j_{m}} \otimes\left(x_{j_{\pi(1)}} \otimes x_{j_{\pi(2)}}-q_{(\pi(1), \pi(2))}^{j_{\pi(1)}, \ldots, j_{\pi(m)}} x_{j_{\pi(2)}} \otimes x_{j_{\pi(1)}}\right) \\
& \quad \otimes x_{j_{\pi(3)}} \otimes \cdots \otimes x_{j_{\pi(m)}} \otimes 1,
\end{aligned}
$$

which is visibly in $A \otimes R \otimes V^{\otimes(m-2)} \otimes A$.

Lemma 2.3. The map $\phi$ defined in equation (3) is a chain map.

Proof. We must show that

$$
\phi_{m-1} \circ d_{m}\left(1^{\otimes 2} \otimes x_{j_{1}} \wedge \cdots \wedge x_{j_{m}}\right)=\delta_{m} \circ \phi_{m}\left(1^{\otimes 2} \otimes x_{j_{1}} \wedge \cdots \wedge x_{j_{m}}\right)
$$

for all $j_{1}, \ldots, j_{m}$ with $j_{1}<\cdots<j_{m}$, where $\delta_{m}$ is the differential on the bar complex (11). This may easily be checked when $m=0$.

By Remark 2.2, the formula (3) for $\phi_{m}$ and the formula for $\delta_{m}$, the right side of (51) is

$$
\sum_{\pi \in S_{m}}(\operatorname{sgn} \pi) q_{\pi}^{j_{1}, \ldots, j_{m}}\left(x_{j_{\pi(1)}} \otimes \cdots \otimes x_{j_{\pi(m)}} \otimes 1+(-1)^{m} \otimes x_{j_{\pi(1)}} \otimes \cdots \otimes x_{j_{\pi(m)}}\right)
$$


for all $m \geq 1$. The left side of (5) is

$$
\begin{aligned}
& \phi_{m-1}\left(\sum_{i=1}^{m}(-1)^{i+1}\left(\left(\prod_{s=1}^{i} q_{j_{s}, j_{i}}\right) x_{j_{i}} \otimes 1-\left(\prod_{s=i}^{m} q_{j_{i}, j_{s}}\right) \otimes x_{j_{i}}\right)\right. \\
& \left.\otimes x_{j_{1}} \wedge \cdots \wedge \hat{x}_{j_{i}} \wedge \cdots \wedge x_{j_{m}}\right) \\
& =\sum_{i=1}^{m}(-1)^{i+1}\left(\left(\prod_{s=1}^{i} q_{j_{s}, j_{i}}\right) x_{j_{i}} \otimes 1-\left(\prod_{s=i}^{m} q_{j_{i}, j_{s}}\right) \otimes x_{j_{i}}\right) \\
& \otimes\left(\sum_{\pi^{\prime} \in S_{m-1}^{i}}\left(\operatorname{sgn} \pi^{\prime}\right) q_{\pi^{\prime}}^{j_{1}, \ldots, \hat{j}_{i}, \ldots, j_{m}} x_{j_{\pi^{\prime}(1)}} \otimes \cdots \otimes x_{j_{\pi^{\prime}(m)}}\right) \\
& =\sum_{i=1}^{m}(-1)^{i+1} \sum_{\pi^{\prime} \in S_{m-1}^{i}}\left(\left(\operatorname{sgn} \pi^{\prime}\right)\left(\prod_{s=1}^{i} q_{j_{s}, j_{i}}\right) q_{\pi^{\prime}}^{j_{1}, \ldots, \hat{j}_{i}, \ldots, j_{m}} x_{j_{i}} \otimes x_{j_{\pi^{\prime}(1)}} \otimes \cdots \otimes x_{j_{\pi^{\prime}(m)}} \otimes 1\right. \\
& \left.-\left(\operatorname{sgn} \pi^{\prime}\right)\left(\prod_{s=i}^{m} q_{j_{i}, j_{s}}\right) q_{\pi^{\prime}}^{j_{1}, \ldots, \hat{j}_{i}, \ldots, j_{m}} \otimes x_{j_{\pi^{\prime}(1)}} \otimes \cdots \otimes x_{j_{\pi^{\prime}(m)}} \otimes x_{j_{i}}\right)
\end{aligned}
$$

where for each $i, S_{m-1}^{i}$ denotes the symmetric group on $\{1, \ldots, \hat{i}, \ldots, m\}$. In the first set of summands above, if $\pi:=\pi^{\prime}(i, i-1, \ldots, 1)$, then $\operatorname{sgn} \pi^{\prime}=(-1)^{i+1} \operatorname{sgn} \pi$, and we may replace $\left(\operatorname{sgn} \pi^{\prime}\right) x_{j_{i}} \otimes x_{j_{\pi^{\prime}(1)}} \otimes \cdots \otimes x_{j_{\pi^{\prime}(m)}} \otimes 1$ by $(-1)^{i-1}(\operatorname{sgn} \pi) x_{j_{\pi(1)}} \otimes$ $\cdots \otimes x_{j_{\pi(m)}} \otimes 1$. Similarly, in the second set of summands, let $\pi=\pi^{\prime}(i, i+1$, $\ldots, m)$, and replace $\left(\operatorname{sgn} \pi^{\prime}\right) \otimes x_{j_{\pi^{\prime}(1)}} \otimes \cdots \otimes x_{j_{\pi^{\prime}(m)}} \otimes x_{j_{i}}$ by $(-1)^{m-i}(\operatorname{sgn} \pi) \otimes$ $x_{j_{\pi(1)}} \otimes \cdots \otimes x_{j_{\pi(m)}}$. Again, for the first set of summands, notice that $q_{\pi}^{j_{1}, \ldots, j_{m}}=$ $\left(\prod_{s=1}^{i} q_{j_{s}, j_{i}}\right) q_{\pi^{\prime}}^{j_{1}, \ldots, \hat{j}_{i}, \ldots, j_{m}}$, and for the second set $q_{\pi}^{j_{1}, \ldots, j_{m}}=\left(\prod_{s=i}^{m} q_{j_{i}, j_{s}}\right) q_{\pi^{\prime}}^{j_{1}, \ldots, \hat{j_{i}, \ldots, j_{m}}}$. Making all such replacements, we find that the left side of (5) is indeed equal to the right side.

Remark 2.4. The image of $\phi_{m}$ is a free $A^{e}$-submodule of $A^{\otimes(m+2)}$ that is a direct summand of $A^{\otimes(m+2)}$ as an $A^{e}$-module: Take a vector space complement in $\mathbb{k} \otimes$ $V^{\otimes m} \otimes \mathbb{k}$ to the image of $\mathbb{k} \otimes \mathbb{k} \otimes \bigwedge^{m}(V)$ under $\phi_{m}$ and extend to the required complementary $A^{e}$-module direct summand of $A^{\otimes(m+2)}$. It follows that there is a chain map $\psi$ from the bar resolution to $A^{e} \otimes \Lambda^{\bullet}(V)$ for which $\psi \phi$ is the identity map.

\section{THE CUP PRODUCT}

As before, let $A$ denote the quantum symmetric algebra $S_{\mathbf{q}}(V)$ and let $A^{e}$ denote the enveloping algebra $A \otimes A^{\text {op }}$. Let $B$ denote an $A$-bimodule. In this section, we will describe the cup product on Hochschild cohomology $\operatorname{HH}^{*}(A, B)=\operatorname{Ext}_{A^{e}}(A, B)$, when $B$ additionally has a compatible algebra structure.

We begin by applying $\operatorname{Hom}_{A^{e}}(\cdot, B)$ to (2), dropping the term $\operatorname{Hom}_{A^{e}}(A, B)$ to obtain the complex

(6)

$0 \rightarrow \operatorname{Hom}_{A^{e}}\left(A^{e}, B\right) \stackrel{\tilde{d}_{1}}{\longrightarrow} \operatorname{Hom}_{A^{e}}\left(A^{e} \otimes \bigwedge^{1}(V), B\right) \stackrel{\tilde{d}_{2}}{\longrightarrow} \operatorname{Hom}_{A^{e}}\left(A^{e} \otimes \bigwedge^{2}(V), B\right) \rightarrow \cdots$. 
That is, the degree $m$ term is $\operatorname{Hom}_{A^{e}}\left(A^{e} \otimes \bigwedge^{m}(V), B\right)$, and $\tilde{d}_{m}$ is defined by $\tilde{d}_{m}(F)=F \circ d_{m}$, for all $F \in \operatorname{Hom}_{A^{e}}\left(A^{e} \otimes \bigwedge^{m-1}(V), B\right)$.

We identify the spaces $\operatorname{Hom}_{A^{e}}\left(A^{e} \otimes \bigwedge^{m}(V), B\right)$ and $B \otimes \bigwedge^{m}\left(V^{*}\right)$ via the map

$$
\begin{aligned}
& \Theta_{m}: \operatorname{Hom}_{A^{e}}\left(A^{e} \otimes \bigwedge^{m}(V), B\right) \rightarrow B \otimes \bigwedge^{m}\left(V^{*}\right), \\
& F \mapsto \sum_{\substack{\beta \in\{0,1\}^{N} \\
|\beta|=m}} F\left(1^{\otimes 2} \otimes x^{\wedge \beta}\right) \otimes\left(x^{*}\right)^{\wedge \beta} .
\end{aligned}
$$

We thus obtain the following complex, which is equivalent to the complex in (6):

$$
0 \rightarrow B \stackrel{d_{1}^{*}}{\longrightarrow} B \otimes \bigwedge^{1}\left(V^{*}\right) \stackrel{d_{2}^{*}}{\longrightarrow} B \otimes \bigwedge^{2}\left(V^{*}\right) \rightarrow \cdots .
$$

That is, the degree $m$ term is $B \otimes \bigwedge^{m}\left(V^{*}\right)$, and $d_{m}^{*}$ is defined by $d_{m}^{*}=\Theta_{m} \circ \tilde{d}_{m} \circ$ $\Theta_{m-1}^{-1}$, where $\Theta_{0}^{-1}$ takes $b \in B$ to $F \in \operatorname{Hom}_{A^{e}}\left(A^{e}, B\right)$ defined by $F\left(1^{\otimes 2}\right)=b$.

Let us describe $d_{m}^{*}$ explicitly. For any $b \in B$ and $\beta \in\{0,1\}^{N}$ with $|\beta|=m-1$ we have

$$
\begin{aligned}
& d_{m}^{*}\left(b \otimes\left(x^{*}\right)^{\wedge \beta}\right) \\
&=\left(\Theta_{m} \circ \tilde{d}_{m} \circ \Theta_{m-1}^{-1}\right)\left(b \otimes\left(x^{*}\right)^{\wedge \beta}\right) \\
&= \Theta_{m}\left(\Theta_{m-1}^{-1}\left(b \otimes\left(x^{*}\right)^{\wedge \beta}\right) \circ d_{m}\right) \\
&= \sum_{\substack{\beta^{\prime} \in\{0,1\}^{N} \\
\left|\beta^{\prime}\right|=m}}\left(\Theta_{m-1}^{-1}\left(b \otimes\left(x^{*}\right)^{\wedge \beta}\right) \circ d_{m}\right)\left(1^{\otimes 2} \otimes x^{\wedge \beta^{\prime}}\right) \otimes\left(x^{*}\right)^{\wedge \beta^{\prime}} \\
&= \sum_{i=1}^{N} \delta_{\beta_{i}, 0}\left(\Theta_{m-1}^{-1}\left(b \otimes\left(x^{*}\right)^{\wedge \beta}\right) \circ d_{m}\right)\left(1^{\otimes 2} \otimes x^{\wedge(\beta+[i])}\right) \otimes\left(x^{*}\right)^{\wedge(\beta+[i])} \\
&= \sum_{i=1}^{N} \delta_{\beta_{i}, 0}\left(\Theta_{m-1}^{-1}\left(b \otimes\left(x^{*}\right)^{\wedge \beta}\right)\right) \\
&\left((-1)^{\sum_{s=1}^{i} \beta_{s}}\left[\left(\prod_{s=1}^{i} q_{s, i}^{\beta_{s}}\right) x_{i} \otimes 1-\left(\prod_{s=i}^{N} q_{i, s}^{\beta_{s}}\right) \otimes x_{i}\right] \otimes x^{\wedge \beta}\right) \otimes\left(x^{*}\right)^{\wedge(\beta+[i])} .
\end{aligned}
$$

Using the $A^{e}$-linearity of $\Theta_{m-1}^{-1}\left(b \otimes\left(x^{*}\right)^{\wedge \beta}\right)$ and the definition of $\Theta$, the above expression may be rewritten to show that $d_{m}^{*}\left(b \otimes\left(x^{*}\right)^{\wedge \beta}\right)$ is equal to

$$
\sum_{i=1}^{N} \delta_{\beta_{i}, 0}(-1)^{\sum_{s=1}^{i} \beta_{s}}\left[\left(\prod_{s=1}^{i} q_{s, i}^{\beta_{s}}\right) x_{i} b-\left(\prod_{s=i}^{N} q_{i, s}^{\beta_{s}}\right) b x_{i}\right] \otimes\left(x^{*}\right)^{\wedge(\beta+[i])} .
$$

We will use these expressions for the differentials in the sequel.

Let $\mathbf{q}$ be a tuple of scalars as in the introduction. We define the quantum exterior algebra

$$
\bigwedge_{\mathbf{q}}(V)=T(V) /\left(x_{i} x_{j}+q_{i, j} x_{j} x_{i} \mid 1 \leq i, j \leq N\right),
$$

where $T(V)$ is the tensor algebra on $V$. Note that the relations corresponding to $i=j$ are $2 x_{i}^{2}=0$ since $q_{i i}=1$; that is, $x_{i}^{2}=0$ in $\bigwedge_{\mathbf{q}}(V)$. It follows that the dimension of $\bigwedge_{\mathbf{q}}(V)$ as a (graded) vector space is the same as that of the exterior algebra $\bigwedge(V)$.

Let $\mathbf{q}^{-1}$ denote the tuple consisting of all inverses of components of $\mathbf{q}$, that is, $\mathbf{q}^{-1}=\left\{q_{i, j}^{-1}\right\}_{1 \leq i, j \leq N}$. Using this notation, we have the following cup product 
theorem. Our main applications are to the cases $B=S_{\mathbf{q}}(V)$ and $B=S_{\mathbf{q}}(V) \rtimes G$ for some finite group $G$ in the next section.

Theorem 3.1. Let $B$ be an $S_{\mathbf{q}}(V)$-bimodule algebra. Then the Hochschild cohomology algebra $\mathrm{HH}^{*}\left(S_{\mathbf{q}}(V), B\right)$ is a subquotient algebra of the tensor product $B \otimes \bigwedge_{\mathbf{q}^{-1}}\left(V^{*}\right)$. That is, the cup product on $\mathrm{HH}^{*}\left(S_{\mathbf{q}}(V), B\right)$ descends from the product on $B \otimes \bigwedge_{\mathbf{q}^{-1}}\left(V^{*}\right)$.

Proof. Letting $A=S_{\mathbf{q}}(V)$, by Remark 2.4 there is a chain map $\psi: A^{\otimes(m+2)} \rightarrow$ $A^{e} \otimes \bigwedge^{m}(V)$ such that $\psi \phi$ is the identity map. (We will not need an explicit formula for $\psi$.)

We will use the cup product as defined on the bar complex, together with the chain maps $\phi$ and $\psi$ : For two cocycles $\mu$ and $\nu$ defined on $A^{e} \otimes \Lambda(V)$, in degrees $m$ and $n$ respectively, their cup product is given by

$$
\phi^{*}\left(\psi^{*}(\mu) \smile \psi^{*}(\nu)\right) .
$$

This function is defined by its action on all elements of the form $1^{\otimes 2} \otimes x_{j_{1}} \wedge \cdots \wedge$ $x_{j_{m+n}}$, which we calculate next. Let $S_{m, n}$ denote the set of all $m, n$-shuffles, that is, all $\rho \in S_{m+n}$ for which $\rho(1)<\cdots<\rho(m)$ and $\rho(m+1)<\cdots<\rho(m+n)$. Note that these shuffles form a set of coset representatives of the subgroup $S_{m} \times S_{n}$ of $S_{m+n}$, where $S_{m}$ acts on $\{1, \ldots, m\}$ and $S_{n}$ acts on $\{m+1, \ldots, m+n\}$. Thus we may write each $\pi \in S_{m+n}$ as $\pi=\rho \sigma \tau$, where $\rho \in S_{m, n}, \sigma \in S_{m}, \tau \in S_{n}$. By Lemma 2.1] writing $\rho \sigma \tau=\left(\rho \sigma \rho^{-1}\right)\left(\rho \tau \rho^{-1}\right) \rho$, we have

$$
\begin{aligned}
& \phi^{*}\left(\psi^{*}(\mu) \smile \psi^{*}(\nu)\right)\left(1^{\otimes 2} \otimes x_{j_{1}} \wedge \cdots \wedge x_{j_{m+n}}\right) \\
= & \left(\psi^{*}(\mu) \smile \psi^{*}(\nu)\right)\left(\sum_{\pi \in S_{m+n}}(\operatorname{sgn} \pi) q_{\pi}^{j_{1}, \ldots, j_{m+n}} \otimes x_{j_{\pi(1)}} \otimes \cdots \otimes x_{j_{\pi(m+n)}} \otimes 1\right) \\
= & \sum_{\pi \in S_{m+n}}(\operatorname{sgn} \pi) q_{\pi}^{j_{1}, \ldots, j_{m+n}} \psi^{*}(\mu)\left(1 \otimes x_{j_{\pi(1)}} \otimes \cdots \otimes x_{j_{\pi(m)}} \otimes 1\right) \\
& \cdot \psi^{*}(\nu)\left(1 \otimes x_{j_{\pi(m+1)}} \otimes \cdots \otimes x_{j_{\pi(m+n)}} \otimes 1\right) \\
= & \sum_{\rho \in S_{m, n}} \sum_{\sigma \in S_{m}, \tau \in S_{n}}(\operatorname{sgn} \rho \sigma \tau) q_{\rho \sigma \tau}^{j_{1}, \ldots, j_{m+n}} \psi^{*}(\mu)\left(1 \otimes x_{j_{\rho \sigma \tau(1)}} \otimes \cdots \otimes x_{j_{\rho \sigma \tau(m)}} \otimes 1\right) \\
= & \psi_{\rho \in S_{m, n}}(\nu)\left(1 \otimes x_{j_{\rho \sigma \tau(m+1)}} \otimes \cdots \otimes x_{j_{\rho \sigma \tau(m+n)}} \otimes 1\right) \\
& \cdot\left(\sum_{\sigma \in S_{m}}(\operatorname{sgn} \rho) q_{\rho}^{j_{1}, \ldots, j_{m+n}}\right. \\
& \cdot\left(\sum_{\tau \in S_{n}}(\operatorname{sgn} \tau) q_{\rho \sigma \rho^{-1}}^{j_{\rho(1)}, \ldots, j_{\rho(m)}} \psi^{*}(\mu)\left(1 \otimes x_{\rho \tau j^{-1}}^{j_{\rho(m+1)}} \otimes \cdots, j_{\rho(m+n)} \psi^{*}(\nu)\left(1 \otimes x_{j_{\rho \tau(m+1)}} \otimes \cdots \otimes x_{j_{\rho \sigma(m)}} \otimes 1\right)\right)\right. \\
= & \sum_{\rho \in S_{m, n}}(\operatorname{sgn} \rho) q_{\rho}^{j_{1}, \ldots, j_{m+n}} \phi^{*} \psi^{*}(\mu)\left(1^{\otimes 2} \otimes x_{j_{\rho(1)}} \wedge \cdots \wedge x_{j_{\rho(m)}}\right) \\
& \cdot \phi^{*} \psi^{*}(\nu)\left(1^{\otimes 2} \otimes x_{j_{\rho(m+1)}} \wedge \cdots \wedge x_{j_{\rho(m+n)}}\right) .
\end{aligned}
$$

Since $\psi \phi$ is the identity map, $\phi^{*} \psi^{*}(\mu)=\mu$ and $\phi^{*} \psi^{*}(\nu)=\nu$. Now replacing $\mu$ by $b \otimes\left(x^{*}\right)^{\wedge \beta}$ and $\nu$ by $b^{\prime} \otimes\left(x^{*}\right)^{\wedge \beta^{\prime}}$, where $b, b^{\prime} \in B$, we see that only one summand in 
the above can be nonzero and that is the summand corresponding to $\rho$ for which

$$
x^{\wedge \beta}=x_{j_{\rho(1)}} \wedge \cdots \wedge x_{j_{\rho(m)}} \quad \text { and } \quad x^{\wedge \beta^{\prime}}=x_{j_{\rho(m+1)}} \wedge \cdots \wedge x_{j_{\rho(m+n)}} .
$$

For this summand, we obtain $(\operatorname{sgn} \rho) q_{\rho}^{j_{1}, \cdots, j_{m+n}} b b^{\prime}$. Comparing, we also find that

$$
\left(b b^{\prime} \otimes\left(x^{*}\right)^{\wedge \beta} \wedge\left(x^{*}\right)^{\wedge \beta^{\prime}}\right)\left(1^{\otimes 2} \otimes x_{j_{1}} \wedge \cdots \wedge x_{j_{m+n}}\right)=(\operatorname{sgn} \rho) q_{\rho}^{j_{1}, \cdots, j_{m+n}} b b^{\prime},
$$

by permuting the exterior factors in $\left(x^{*}\right)^{\wedge \beta} \wedge\left(x^{*}\right)^{\wedge \beta^{\prime}}$ via $\rho$ before applying the

function and by using the identity $q_{\rho^{-1}}^{j_{\rho(1)}, \ldots, j_{\rho(m+n)}}=\left(q_{\rho}^{j_{1}, \ldots, j_{m+n}}\right)^{-1}$ (a consequence of Lemma 2.1). Therefore the product is as claimed.

Remark 3.2. It is not necessary that the characteristic of $\mathbb{k}$ be 0 for Theorem 3.1] While Wambst's proof that complex (2) is exact requires characteristic 0, a similar resolution may be obtained in positive characteristic by using a twisted tensor product construction [B] or general theory for Koszul algebras [BG]. Our proof of Theorem 3.1] uses only resolution (2) and its embedding into the bar resolution.

\section{The Hochschild cohomology algebra of $S_{\mathbf{q}}(V) \rtimes G$}

Let $G$ be a finite group acting on $A=S_{\mathbf{q}}(V)$ by graded algebra automorphisms. We are interested in the cohomology of the skew group algebra $A \rtimes G$. Since the characteristic of $\mathbb{k}$ is zero, this is known to be isomorphic to the $G$-invariant subalgebra of the cohomology $\operatorname{HH}^{*}(A, A \rtimes G)$ of $A$ with coefficients in $A \rtimes G$. (See for example [S.) In this section we will compute this latter cohomology, $\mathrm{HH}^{*}(A, A \rtimes G)=\operatorname{Ext}_{A^{e}}(A, A \rtimes G)$, in the case when $G$ acts diagonally on the basis $x_{1}, \ldots, x_{N}$ of $V$. Note that each $g$-component $A_{g}$ is a (left) $A^{e}$-module (see Section 2). Also note that since $A \rtimes G=\bigoplus_{g \in G} A_{g}$ as an $A^{e}$-module,

$$
\operatorname{Ext}_{A^{e}}(A, A \rtimes G) \cong \bigoplus_{g \in G} \operatorname{Ext}_{A^{e}}\left(A, A_{g}\right) .
$$

We will compute the summands $\operatorname{Ext}_{A^{e}}\left(A, A_{g}\right)$.

Fix $g \in G$. In Section 3 we applied the Hom functor $\operatorname{Hom}_{A^{e}}(\cdot, B)$, for any $A$ bimodule $B$, to the $A^{e}$-resolution of $A$ in (2), and we made appropriate identification to obtain the complex (17). When we specialize this complex to $B=A_{g}$, we obtain

$$
0 \rightarrow A_{g} \stackrel{d_{1}^{*}}{\longrightarrow} A_{g} \otimes \bigwedge^{1}\left(V^{*}\right) \stackrel{d_{2}^{*}}{\longrightarrow} A_{g} \otimes \bigwedge^{2}\left(V^{*}\right) \rightarrow \cdots,
$$

where formula (8) yields the fact that $d_{m}^{*}\left((a \# g) \otimes\left(x^{*}\right)^{\wedge \beta}\right)$ is equal to

$$
\sum_{i=1}^{N} \delta_{\beta_{i}, 0}(-1)^{\sum_{s=1}^{i} \beta_{s}}\left[\left(\left(\prod_{s=1}^{i} q_{s, i}^{\beta_{s}}\right) x_{i} a-\left(\prod_{s=i}^{N} q_{i, s}^{\beta_{s}}\right) a\left({ }^{g} x_{i}\right)\right) \# g\right] \otimes\left(x^{*}\right)^{\wedge(\beta+[i])},
$$

for all $a \in A$ and $\beta \in\{0,1\}^{N}$ with $|\beta|=m-1$.

4.1. Additive structure. Suppose $G$ acts diagonally on the basis $x_{1}, \ldots, x_{N}$ of $V$. That is, there exist scalars $\lambda_{g, i} \in \mathbb{k}$ such that ${ }^{g} x_{i}=\lambda_{g, i} x_{i}$ for all $g \in G, i \in$ $\{1, \ldots, N\}$.

For each $g \in G$, define

$$
C_{g}:=\left\{\gamma \in(\mathbb{N} \cup\{-1\})^{N} \mid \text { for each } i \in\{1, \ldots, N\}, \prod_{s=1}^{N} q_{i, s}^{\gamma_{s}}=\lambda_{g, i} \text { or } \gamma_{i}=-1\right\} .
$$


The following theorem gives the structure of the Hochschild cohomology as a graded vector space. Recall definition (9) of the quantum exterior algebra.

Theorem 4.1. If $G$ acts diagonally on the chosen basis of $V$, then we have that $\mathrm{HH}^{*}\left(S_{\mathbf{q}}(V), S_{\mathbf{q}}(V)_{g}\right)$ is the graded vector subspace of $S_{\mathbf{q}}(V)_{g} \otimes \bigwedge_{\mathbf{q}^{-1}}\left(V^{*}\right)$ given by

$$
\mathrm{HH}^{m}\left(S_{\mathbf{q}}(V), S_{\mathbf{q}}(V)_{g}\right) \cong \bigoplus_{\substack{\beta \in\{0,1\}^{N} \\|\beta|=m}} \bigoplus_{\substack{\alpha \in \mathbb{N}^{N} \\ \alpha-\beta \in C_{g}}} \operatorname{span}_{\mathbb{k}}\left\{\left(x^{\alpha} \# g\right) \otimes\left(x^{*}\right)^{\wedge \beta}\right\},
$$

for all $m \in \mathbb{N}, g \in G$. Therefore,

$$
\operatorname{HH}^{m}\left(S_{\mathbf{q}}(V), S_{\mathbf{q}}(V) \rtimes G\right) \cong \bigoplus_{g \in G} \bigoplus_{\substack{\beta \in\{0,1\}^{N} \\|\beta|=m}} \bigoplus_{\substack{\alpha \in \mathbb{N}^{N} \\ \alpha-\beta \in C_{g}}} \operatorname{span}_{\mathbb{k}}\left\{\left(x^{\alpha} \# g\right) \otimes\left(x^{*}\right)^{\wedge \beta}\right\},
$$

for all $m \in \mathbb{N}$, and $\mathrm{HH}^{m}\left(S_{\mathbf{q}}(V) \rtimes G\right)$ is its $G$-invariant subspace.

Example 4.2. Fix $g \in G$. We wish to describe $\mathrm{HH}_{g}^{*}:=\mathrm{HH}^{\bullet}\left(S_{\mathbf{q}}(V), S_{\mathbf{q}}(V)_{g}\right)$ when $N=2$. We will work out two special cases. When $q_{1,2}$ is not a root of unity and $\lambda_{g, 1}, \lambda_{g, 2}$ are not both equal to 1 , we have

$$
\begin{aligned}
& \mathrm{HH}_{g}^{0} \cong\{0\}, \\
& \mathrm{HH}_{g}^{1} \cong\{0\}, \\
& \mathrm{HH}_{g}^{2} \cong \operatorname{span}_{\mathbb{k}}\left\{(1 \# g) \otimes x_{1}^{*} \wedge x_{2}^{*}\right\} .
\end{aligned}
$$

For the second case, assume $q_{1,2}$ is simultaneously a primitive $\ell$ th root of unity, a $\ell_{1}$ th root of $\lambda_{g, 1}$, and a $\ell_{2}$ th root of $\lambda_{g, 2}^{-1}$. Also assume $q_{1,2} \neq \lambda_{g, 2}$ and $q_{1,2}^{-1} \neq \lambda_{g, 1}$. Then we have

$$
\begin{aligned}
& \mathrm{HH}_{g}^{0} \cong \operatorname{span}_{\mathbb{k}}\left\{x_{1}^{\alpha_{1}} x_{2}^{\alpha_{2}} \# g \mid \alpha_{1}, \alpha_{2} \in \mathbb{N}, \ell \text { divides both } \alpha_{1}-\ell_{2} \text { and } \alpha_{2}-\ell_{1}\right\}, \\
& H_{g}^{1} \cong \operatorname{span}_{\mathbb{k}}\left\{\left(x_{1}^{\alpha_{1}} x_{2}^{\alpha_{2}} \# g\right) \otimes x_{1}^{*} \mid \alpha_{1}, \alpha_{2} \in \mathbb{N}, \ell \text { divides both } \alpha_{2}-\ell_{1}\right.\left.\quad \text { and } \alpha_{1}-\ell_{2}-1\right\} \\
& \bigoplus \operatorname{span}_{\mathbb{k}}\left\{\left(x_{1}^{\alpha_{1}} x_{2}^{\alpha_{2}} \# g\right) \otimes x_{2}^{*} \mid \alpha_{1}, \alpha_{2} \in \mathbb{N}, \ell \text { divides both } \alpha_{1}-\ell_{2}\right. \\
&\left.\quad \text { and } \alpha_{2}-\ell_{1}-1\right\}, \\
& H_{g}^{2} \cong \operatorname{span}_{\mathbb{k}}\left\{(1 \# g) \otimes x_{1}^{*} \wedge x_{2}^{*}\right\} \\
& \bigoplus \operatorname{span}_{\mathbb{k}}\left\{\left(x_{1}^{\alpha_{1}} x_{2}^{\alpha_{2}} \# g\right) \otimes x_{1}^{*} \wedge x_{2}^{*} \mid \alpha_{1}, \alpha_{2} \in \mathbb{N}, \ell \text { divides } \alpha_{1}-\ell_{2}-1\right. \\
&\left.\quad \text { and } \alpha_{2}-\ell_{1}-1\right\} .
\end{aligned}
$$

Thus $\mathrm{HH}_{g}^{0}$ is the free module over $Z\left(S_{\mathbf{q}}(V)\right)$ generated by $x_{1}^{\ell_{2}} x_{2}^{\ell_{1}} \# g, \mathrm{HH}_{g}^{1}$ is the free $Z\left(S_{\mathbf{q}}(V)\right)$-module generated by $\left(x_{1}^{\ell_{2}+1} x_{2}^{\ell_{1}} \# g\right) \otimes x_{1}^{*}$ and $\left(x_{1}^{\ell_{2}} x_{2}^{\ell_{1}+1} \# g\right) \otimes x_{2}^{*}$, and $\mathrm{HH}_{g}^{2}$ is the direct sum of the $\mathbb{k}$-linear span of $(1 \# g) \otimes x_{1}^{*} \wedge x_{2}^{*}$ and the free $Z\left(S_{\mathbf{q}}(V)\right)$-module generated by $\left(x_{1}^{\ell_{2}+1} x_{2}^{\ell_{1}+1} \# g\right) \otimes x_{1}^{*} \wedge x_{2}^{*}$.

Define

$$
C:=\left\{\gamma \in(\mathbb{N} \cup\{-1\})^{N} \mid \text { for each } i \in\{1, \ldots, N\}, \prod_{s=1}^{N} q_{i, s}^{\gamma_{s}}=1 \text { or } \gamma_{i}=-1\right\} .
$$

Taking $G$ to be the trivial group with one element, we immediately obtain the following: 
Corollary 4.3. $\mathrm{HH}^{*}\left(S_{\mathbf{q}}(V)\right)$ is the graded vector subspace of $S_{\mathbf{q}}(V) \otimes \bigwedge_{\mathbf{q}^{-1}}\left(V^{*}\right)$ given by

$$
\operatorname{HH}^{m}\left(S_{\mathbf{q}}(V)\right) \cong \bigoplus_{\substack{\beta \in\{0,1\}^{N} \\|\beta|=m}} \bigoplus_{\substack{\alpha \in \mathbb{N}^{N} \\ \alpha-\beta \in C}} \operatorname{span}_{\mathbb{k}}\left\{x^{\alpha} \otimes\left(x^{*}\right)^{\wedge \beta}\right\},
$$

for all $m \in \mathbb{N}$.

Example 4.4. When $N=2$, the description of $\mathrm{HH}^{*}\left(S_{\mathbf{q}}(V)\right)$ simplifies considerably. In this case, if $q_{1,2}$ is not a root of unity, then

$$
\begin{aligned}
& \operatorname{HH}^{0}\left(S_{\mathbf{q}}(V)\right) \cong \mathbb{k}, \\
& \operatorname{HH}^{1}\left(S_{\mathbf{q}}(V)\right) \cong \operatorname{span}_{\mathbb{k}}\left\{x_{1} \otimes x_{1}^{*}, x_{2} \otimes x_{2}^{*}\right\}, \\
& \mathrm{HH}^{2}\left(S_{\mathbf{q}}(V)\right) \cong \operatorname{span}_{\mathbb{k}}\left\{1 \otimes x_{1}^{*} \wedge x_{2}^{*}, x_{1} x_{2} \otimes x_{1}^{*} \otimes x_{2}^{*}\right\},
\end{aligned}
$$

and if $q_{1,2}$ is a primitive $\ell$ th root of unity, $\ell \geq 2$, then

$$
\begin{aligned}
& \mathrm{HH}^{0}\left(S_{\mathbf{q}}(V)\right) \cong \operatorname{span}_{\mathbb{k}}\left\{x_{1}^{\alpha_{1}} x_{2}^{\alpha_{2}} \mid \alpha_{1}, \alpha_{2} \in \mathbb{N}, \ell \text { divides both } \alpha_{1} \text { and } \alpha_{2}\right\}, \\
& \mathrm{HH}^{1}\left(S_{\mathbf{q}}(V)\right) \cong \operatorname{span}_{\mathbb{k}}\left\{x_{1}^{\alpha_{1}} x_{2}^{\alpha_{2}} \otimes x_{1}^{*} \mid \alpha_{1}, \alpha_{2} \in \mathbb{N}, \ell \text { divides both } \alpha_{1}-1 \text { and } \alpha_{2}\right\} \\
& \bigoplus \operatorname{span}_{\mathbb{k}}\left\{x_{1}^{\alpha_{1}} x_{2}^{\alpha_{2}} \otimes x_{2}^{*} \mid \alpha_{1}, \alpha_{2} \in \mathbb{N}, \ell \text { divides both } \alpha_{1}\right. \\
&\text { and } \left.\alpha_{2}-1\right\}, \\
& \mathrm{HH}^{2}\left(S_{\mathbf{q}}(V)\right) \cong \operatorname{span}_{\mathbb{k}}\left\{1 \otimes x_{1}^{*} \wedge x_{2}^{*}\right\}, \\
& \bigoplus \operatorname{span}_{\mathbb{k}}\left\{x_{1}^{\alpha_{1}} x_{2}^{\alpha_{2}} \otimes x_{1}^{*} \wedge x_{2}^{*} \mid \alpha_{1}, \alpha_{2} \in \mathbb{N}, \ell \text { divides } \alpha_{1}-1\right. \\
&\text { and } \left.\alpha_{2}-1\right\} .
\end{aligned}
$$

Note that in the first case, the center of $S_{\mathbf{q}}(V)$ is $Z\left(S_{\mathbf{q}}(V)\right)=\mathbb{k}$, while in the second case, $Z\left(S_{\mathbf{q}}(V)\right)$ is generated by $x_{1}^{\ell}$ and $x_{2}^{\ell}$. Thus in either case, $\operatorname{HH}^{0}\left(S_{\mathbf{q}}(V)\right)=$ $Z\left(S_{\mathbf{q}}(V)\right)$ as expected, $\operatorname{HH}^{1}\left(S_{\mathbf{q}}(V)\right)$ is the free $Z\left(S_{\mathbf{q}}(V)\right)$-module generated by $x_{1} \otimes$ $x_{1}^{*}$ and $x_{2} \otimes x_{2}^{*}$, and $\mathrm{HH}^{2}\left(S_{\mathbf{q}}(V)\right)$ is the direct sum of the $\mathbb{k}$-linear span of $1 \otimes x_{1}^{*} \wedge x_{2}^{*}$ and the free $Z\left(S_{\mathbf{q}}(V)\right)$-module generated by $x_{1} x_{2} \otimes x_{1}^{*} \wedge x_{2}^{*}$. Similar expressions may be obtained when $N \geq 3$, but there are more cases to consider due to the many more parameters involved.

We introduce some notation and lemmas before proving Theorem 4.1

Fix $g \in G$. For any $\alpha \in \mathbb{N}^{N}, \beta \in\{0,1\}^{N}$, and $i \in\{1, \ldots, N\}$, define

$$
\Omega_{g}(\alpha, \beta, i):=\left\{\begin{array}{l}
0, \text { if } \prod_{s=1}^{N} q_{i, s}^{\alpha_{s}-\beta_{s}}=\lambda_{g, i}, \\
0, \text { if } \beta_{i}=1, \\
\varepsilon(\beta, i)\left(\prod_{s=1}^{i} q_{i, s}^{\alpha_{s}-\beta_{s}}-\lambda_{g, i} \prod_{s=i}^{N} q_{s, i}^{\alpha_{s}-\beta_{s}}\right), \text { otherwise, }
\end{array}\right.
$$

where $\varepsilon(\beta, i)=(-1)^{\sum_{s=1}^{i} \beta_{s}}$.

Then, using formula (11) for $d_{m}^{*}$ we see that

$$
d_{m}^{*}\left(\left(x^{\alpha} \# g\right) \otimes\left(x^{*}\right)^{\wedge \beta}\right)=\sum_{i=1}^{N} \Omega_{g}(\alpha, \beta, i)\left(x^{\alpha+[i]} \# g\right) \otimes\left(x^{*}\right)^{\wedge(\beta+[i])},
$$

for all $\alpha \in \mathbb{N}^{N}$ and $\beta \in\{0,1\}^{N}$ with $|\beta|=m-1$. 
For any $\gamma \in(\mathbb{N} \cup\{-1\})^{N}$ and $m \in \mathbb{N}$, define

$K_{g, \gamma}^{m}:=\operatorname{span}_{\mathbb{k}}\left\{\left(x^{\alpha} \# g\right) \otimes\left(x^{*}\right)^{\wedge \beta}\left|\alpha \in \mathbb{N}^{N}, \beta \in\{0,1\}^{N},\right| \beta \mid=m\right.$, and $\left.\alpha-\beta=\gamma\right\}$.

Let $K_{g, \gamma}^{\cdot}$ denote the subcomplex of (10) whose $m$ th term is given by $K_{g, \gamma}^{m}$. That $K_{g, \gamma}^{*}$ is indeed a subcomplex of (10) follows from (14). We immediately obtain:

Lemma 4.5. The complex (10) admits a grading by $(\mathbb{N} \cup\{-1\})^{N}$. Precisely, the $m$ th term of complex (10) decomposes as $\bigoplus_{\gamma \in(\mathbb{N} \cup\{-1\})^{N}} K_{g, \gamma}^{m}$, for all $m \in \mathbb{N}$.

We now handle separately the cases where $\gamma$ is or is not in the set $C_{g}$ defined in (12). For the proof of the next lemma, we will need some notation: For any $\gamma \in(\mathbb{N} \cup\{-1\})^{N}$, define

$$
\|\gamma\|_{g}:=\#\left\{i \in\{1, \ldots, N\} \mid \prod_{s=1}^{N} q_{i, s}^{\gamma_{s}} \neq \lambda_{g, i} \text { and } \gamma_{i} \neq-1\right\} .
$$

Lemma 4.6. Let $\gamma \in\left((\mathbb{N} \cup\{-1\})^{N} \backslash C_{g}\right)$. Then, the subcomplex $K_{g, \gamma}^{*}$ of (10) is acyclic.

Proof. We will show that the identity chain map of the complex $K_{g, \gamma}^{*}$ is nullhomotopic. First, define the following scalar. Let $\alpha \in \mathbb{N}^{N}, \beta \in\{0,1\}^{N}$, and $i \in\{1, \ldots N\}$. Define

$$
\omega_{g}(\alpha, \beta, i):=\left\{\begin{array}{l}
0, \text { if } \prod_{s=1}^{N} q_{i, s}^{\alpha_{s}-\beta_{s}}=\lambda_{g, i} \\
0, \text { if } \alpha_{i}=0 \\
0, \text { if } \beta_{i}=0, \\
\Omega_{g}(\alpha-[i], \beta-[i], i)^{-1}, \text { otherwise. }
\end{array}\right.
$$

Now, fix $m \in \mathbb{N}$ and suppose that $|\beta|=m$ and $\alpha-\beta=\gamma$. Define

$$
h_{m}: K_{g, \gamma}^{m} \rightarrow K_{g, \gamma}^{m-1}
$$

by

$$
h_{m}\left(\left(x^{\alpha} \# g\right) \otimes\left(x^{*}\right)^{\wedge \beta}\right):=\frac{1}{\|\gamma\|_{g}} \sum_{i=1}^{N} \omega_{g}(\alpha, \beta, i)\left(x^{\alpha-[i]} \# g\right) \otimes\left(x^{*}\right)^{\wedge(\beta-[i])} .
$$

Note that $\|\gamma\|_{g} \neq 0$, as $\gamma \notin C_{g}$.

We contend that

$$
\left(h_{m+1} \circ d_{m+1}^{*}+d_{m}^{*} \circ h_{m}\right)\left(\left(x^{\alpha} \# g\right) \otimes\left(x^{*}\right)^{\wedge \beta}\right)=\left(x^{\alpha} \# g\right) \otimes\left(x^{*}\right)^{\wedge \beta} .
$$


Note that the lemma is proved if this equality is sustained. The left hand side of this equality is equal to

$$
\begin{aligned}
& \frac{1}{\|\gamma\|_{g}} \sum_{i=1}^{N} \sum_{j=1}^{N} \Omega_{g}(\alpha, \beta, i) \omega_{g}(\alpha+[i], \beta+[i], j)\left(x^{\alpha+[i]-[j]} \# g\right) \otimes\left(x^{*}\right)^{\wedge(\beta+[i]-[j])} \\
& \quad+\frac{1}{\|\gamma\|_{g}} \sum_{j=1}^{N} \sum_{i=1}^{N} \omega_{g}(\alpha, \beta, j) \Omega_{g}(\alpha-[j], \beta-[j], i)\left(x^{\alpha+[i]-[j]} \# g\right) \otimes\left(x^{*}\right)^{\wedge(\beta+[i]-[j])} \\
& =\frac{1}{\|\gamma\|_{g}} \sum_{i=1}^{N}\left[\Omega_{g}(\alpha, \beta, i) \omega_{g}(\alpha+[i], \beta+[i], i)+\omega_{g}(\alpha, \beta, i) \Omega_{g}(\alpha-[i], \beta-[i], i)\right] \\
& \quad \times\left(x^{\alpha} \# g\right) \otimes\left(x^{*}\right)^{\wedge \beta} \\
& \quad+\frac{1}{\|\gamma\|_{g}} \sum_{i \neq j}\left[\Omega_{g}(\alpha, \beta, i) \omega_{g}(\alpha+[i], \beta+[i], j)+\omega_{g}(\alpha, \beta, j) \Omega_{g}(\alpha-[j], \beta-[j], i)\right] \\
& \quad \times\left(x^{\alpha+[i]-[j]} \# g\right) \otimes\left(x^{*}\right)^{\wedge(\beta+[i]-[j]) .}
\end{aligned}
$$

It follows from the definition of $\Omega_{g}$ and $\omega_{g}$ that

$$
\sum_{i=1}^{N} \Omega_{g}(\alpha, \beta, i) \omega_{g}(\alpha+[i], \beta+[i], i)+\omega_{g}(\alpha, \beta, i) \Omega_{g}(\alpha-[i], \beta-[i], i)=\|\gamma\|_{g} .
$$

Therefore, it only remains to show that

$$
\Omega_{g}(\alpha, \beta, i) \omega_{g}(\alpha+[i], \beta+[i], j)+\omega_{g}(\alpha, \beta, j) \Omega_{g}(\alpha-[j], \beta-[j], i)=0
$$

whenever $i \neq j$. To this end, define

$$
\Xi_{g}(i, j):=\Omega_{g}(\alpha, \beta, i) \omega_{g}(\alpha+[i], \beta+[i], j)
$$

and

$$
\Xi_{g}^{\prime}(i, j):=\omega_{g}(\alpha, \beta, j) \Omega_{g}(\alpha-[j], \beta-[j], i) .
$$

Suppose $i \neq j$. Then it is clear from definition of $\Omega_{g}$ and $\omega_{g}$ that $\Xi_{g}(i, j)$ and $\Xi_{g}^{\prime}(i, j)$ are simultaneously zero or nonzero, so we may assume that they are nonzero. In this case, we have

$$
\begin{aligned}
\Xi_{g}(i, j)= & \varepsilon(\beta, i) \varepsilon(\beta+[i]-[j], j)\left(\prod_{s=1}^{i} q_{i, s}^{\alpha_{s}-\beta_{s}}-\lambda_{g, i} \prod_{s=i}^{N} q_{s, i}^{\alpha_{s}-\beta_{s}}\right) \\
& \times\left(\prod_{s=1}^{j} q_{j, s}^{\alpha_{s}-\beta_{s}}-\lambda_{g, j} \prod_{s=j}^{N} q_{s, j}^{\alpha_{s}-\beta_{s}}\right)^{-1}
\end{aligned}
$$

and

$$
\begin{aligned}
\Xi_{g}^{\prime}(i, j)= & \varepsilon(\beta-[j], i) \varepsilon(\beta-[j], j)\left(\prod_{s=1}^{j} q_{j, s}^{\alpha_{s}-\beta_{s}}-\lambda_{g, j} \prod_{s=j}^{N} q_{s, j}^{\alpha_{s}-\beta_{s}}\right)^{-1} \\
& \times\left(\prod_{s=1}^{i} q_{i, s}^{\alpha_{s}-\beta_{s}}-\lambda_{g, i} \prod_{s=i}^{N} q_{s, i}^{\alpha_{s}-\beta_{s}}\right) .
\end{aligned}
$$

Therefore, the desired equality $\Xi_{g}(i, j)=-\Xi_{g}^{\prime}(i, j)$ is equivalent to the equality

$$
\varepsilon(\beta-[j], i) \varepsilon(\beta-[j], j)=-\varepsilon(\beta, i) \varepsilon(\beta+[i]-[j], j),
$$


which may be easily verified using the definition of $\varepsilon$, the corresponding conditions under which $\Omega_{g}$ and $\omega_{g}$ are nonzero, and the condition $i \neq j$.

Proof of Theorem 4.1. Observe that the restriction of $d_{*}^{*}$ to $K_{g, \gamma}^{*}$ is zero for all $\gamma \in C_{g}$. The theorem now follows immediately from Lemmas 4.5 and 4.6 .

4.2. Cup product. Assume that $G$ acts diagonally on the basis $x_{1}, \ldots, x_{N}$ of $V$. The following is immediate from Theorem 3.1 when we put $B=S_{\mathbf{q}}(V) \rtimes G$. Recall the definition of $C_{g}$ in (12) and of $\bigwedge_{\mathbf{q}^{-1}}\left(V^{*}\right)$ given by (9).

Theorem 4.7. $\mathrm{HH}^{*}\left(S_{\mathbf{q}}(V), S_{\mathbf{q}}(V) \rtimes G\right)$ is a subquotient algebra of $\left(S_{\mathbf{q}}(V) \rtimes G\right) \otimes$ $\bigwedge_{\mathbf{q}^{-1}}\left(V^{*}\right)$. Thus the cup product on $\mathrm{HH}^{*}\left(S_{\mathbf{q}}(V), S_{\mathbf{q}}(V) \rtimes G\right)$ is given by

$$
\left(\left(x^{\alpha} \# g\right) \otimes\left(x^{*}\right)^{\wedge \beta}\right) \smile\left(\left(x^{\alpha^{\prime}} \# h\right) \otimes\left(x^{*}\right)^{\wedge \beta^{\prime}}\right)=\left(x^{\alpha} \# g\right)\left(x^{\alpha^{\prime}} \# h\right) \otimes\left(x^{*}\right)^{\wedge \beta} \wedge\left(x^{*}\right)^{\wedge \beta^{\prime}}
$$

for all $g, h \in G, \alpha, \alpha^{\prime} \in \mathbb{N}^{N}$ and $\beta, \beta^{\prime} \in\{0,1\}^{N}$ such that $\alpha-\beta \in C_{g}$ and $\alpha^{\prime}-\beta^{\prime} \in$ $C_{h}$. Moreover $\mathrm{HH}^{*}\left(S_{\mathbf{q}}(V) \rtimes G\right)$ is the $G$-invariant subalgebra of $\mathrm{HH}^{*}\left(S_{\mathbf{q}}(V), S_{\mathbf{q}}(V) \rtimes\right.$ $G)$.

Remark 4.8. Note that the above product is zero when the supports of $\beta$ and $\beta^{\prime}$ intersect nontrivially. Furthermore, we understand the above product to be zero (i.e., a coboundary) if $\alpha+\alpha^{\prime}-\left(\beta+\beta^{\prime}\right)$ is not in $C_{g h}$.

Recall the definition of $C$ in (13). Taking $G$ to be the trivial group with one element, we immediately obtain the following:

Corollary 4.9. $\mathrm{HH}^{*}\left(S_{\mathbf{q}}(V)\right)$ is a subquotient algebra of $S_{\mathbf{q}}(V) \otimes \bigwedge_{\mathbf{q}^{-1}}\left(V^{*}\right)$. Thus the cup product on $\mathrm{HH}^{*}\left(S_{\mathbf{q}}(V)\right)$ is given by

$$
\left(x^{\alpha} \otimes\left(x^{*}\right)^{\wedge \beta}\right) \smile\left(x^{\alpha^{\prime}} \otimes\left(x^{*}\right)^{\wedge \beta^{\prime}}\right)=x^{\alpha} x^{\alpha^{\prime}} \otimes\left(x^{*}\right)^{\wedge \beta} \wedge\left(x^{*}\right)^{\wedge \beta^{\prime}}
$$

for all $\alpha, \alpha^{\prime} \in \mathbb{N}^{N}$ and $\beta, \beta^{\prime} \in\{0,1\}^{N}$ such that $\alpha-\beta \in C$ and $\alpha^{\prime}-\beta^{\prime} \in C$.

Remark 4.10. As before, the above product is zero when the supports of $\beta$ and $\beta^{\prime}$ intersect nontrivially. Furthermore, we understand the above product to be zero (i.e., a coboundary) if $\alpha+\alpha^{\prime}-\left(\beta+\beta^{\prime}\right)$ is not in $C$.

\section{REFERENCES}

[AS] N. Andruskiewitsch and H.-J. Schneider, Pointed Hopf algebras, in: New directions in Hopf algebras, MSRI Publ., 43, Cambridge Univ. Press, Cambridge, 2002. MR1913436 (2003e:16043)

[A] R. Anno, Multiplicative structure on the Hochschild cohomology of crossed product algebras, arXiv:math.QA/0511396.

[BB] Y. Bazlov and A. Berenstein, Noncommutative Dunkl operators and braided Cherednik algebras, Sel. Math. New Ser. 14 (2009), no. 3-4, 325-372. MR.2511188

[BGS] A. Beilinson, V. Ginzburg and V. Soergel, Koszul duality patterns in representation theory, J. Amer. Math. Soc. 9 (1996), no. 2, 473-527. MR1322847 (96k:17010)

[BO] P. A. Bergh and S. Oppermann, Cohomology of twisted tensor products, J. Algebra 320 (2008), no. 8, 3327-3338. MR2450729 (2009f:16012)

[BG] A. Braverman and D. Gaitsgory, Poincaré-Birkhoff-Witt Theorem for quadratic algebras of Koszul type, J. Algebra 181 (1996), no. 2, 315-328. MR 1383469 (96m:16012)

[F] M. Farinati, Hochschild duality, localization, and smash products, J. Algebra 284 (2005), no. 1, 415-434. MR2115022 (2005j:16009)

[G] M. Gerstenhaber, The cohomology structure of an associative ring, Ann. of Math. (2) 78 (1963), no. 2, 267-288. MR0161898 (28:5102) 
[GG] J. A. Guccione and J. J. Guccione, Hochschild and cyclic homology of Ore extensions and some examples of quantum algebras, K-Theory 12 (3) (1997), 259-276. MR.1480592 (98i:16012)

[Gu] A. Guichardet, Homologie de Hochschild des déformations quadratiques d'algébres de polynômes, Comm. Algebra 26 (12) (1998), 4309-4330. MR.1661261 (99m:16010)

[GK] V. Ginzburg and D. Kaledin, Poisson deformations of symplectic quotient singularities, Adv. Math. 286 (2004), no. 1, 1-57. MR2065506 (2005h:32072)

[KKZ] E. Kirkman, J. Kuzmanovich, and J. J. Zhang, Shephard-Todd-Chevalley Theorem for skew polynomial rings, Alg. Rep. Theory 13 (2010), no. 2, 127-158.

[M] Yu. I. Manin, Quantum groups and noncommutative geometry, CRM, Université de Montréal, 1988. MR:1016381 (91e:17001)

[P] S. Priddy, Koszul resolutions, Trans. Amer. Math. Soc. 152 (1970), 39-60. MR0265437 $(42: 346)$

[R] L. Richard, Hochschild homology and cohomology of some classical and quantum noncommutative polynomial algebras, J. Pure Appl. Algebra 187 (2004), 255-294. MR 2027905 (2004j:16012)

[SW] A. V. Shepler and S. Witherspoon, Hochschild cohomology and graded Hecke algebras, Trans. Amer. Math. Soc. 360 (2008), no. 8, 3975-4005. MR2395161 (2009a:16015)

[S] D. Ştefan, Hochschild cohomology on Hopf Galois extensions, J. Pure Appl. Algebra 103 (1995), 221-233. MR1358765 (96h:16013)

[W] M. Wambst, Complexes de Koszul quantiques, Ann. Fourier, 43 (1993), no. 4, 1089-1156. MR.1252939(95a:17023)

Department of Mathematics, Texas A\&M University, College Station, Texas 77843

E-mail address: dnaidu@math.tamu.edu

Department of Mathematics, Texas A\&M University, College Station, Texas 77843

E-mail address: pshroff@math.tamu.edu

Department of Mathematics, Texas A\&M University, College Station, Texas 77843

E-mail address: sjw@math.tamu.edu 